\title{
Применение локально-неравновесной диффузионно-дрейфовой модели Каттанео-Вернотта для описания релаксации фототока в диодных структурах при воздействии субпикосекундных импульсов ионизирующих излучений
}

\author{
(C) А.С. Пузанов ${ }^{1}$, С.В. Оболенский ${ }^{1}$, В.А. Козлов ${ }^{1,2}$ \\ ${ }^{1}$ Нижегородский государственный университет им. Н.И. Лобачевского, \\ 603600 Нижний Новгород, Россия \\ ${ }^{2}$ Институт фризики микроструктур Российской академии наук, \\ 607680 Нижний Новгород, Россия \\ E-mail: aspuzanov@inbox.ru
}

(Получена 25 апреля 2018 г. Принята к печати 7 мая 2018 г.)

\begin{abstract}
Рассмотрен процесс релаксации возбуждений в электронно-дырочной плазме при воздействии ионизирующего излучения с длительностью, меньшей времени релаксации энергии и импульса подвижных носителей заряда. На примере расчета переходных ионизационных процессов в кремниевом диоде Шоттки гипервысоких частот проведено сравнение локально-равновесной и локально-неравновесной моделей переноса носителей заряда. Показано, что локально-неравновесная модель имеет более широкую область применимости для описания быстропротекающих релаксационных процессов.
\end{abstract}

DOI: 10.21883/FTP.2018.11.46586.08

\section{1. Введение}

Одним из практических приложений источников ультракоротких импульсов лазерного излучения длительностью 0.1-10 пс является имитация воздействия тяжелых заряженных частиц на изделия микро- и наноэлектроники $[1,2]$. Моделирование реакции полупроводниковых приборов на такого рода воздействия, как лазерного, так и непосредственно тяжелых заряженных частиц, обычно проводится при помощи диффузионнодрейфовой модели, которая позволяет учесть воздействие ионизирующих и дефектообразующих проникающих излучений с помощью изменения скоростей генерации и рекомбинации носителей заряда, а также их подвижностей и коэффициентов диффузии.

Система уравнений переноса носителей заряда в традиционной диффузионно-дрейфовой модели основана на приближении локального равновесия, которое нарушается при длительности возмущающего воздействия, сравнимого со временем релаксации электронно-дырочной плазмы к равновесному состоянию. Следует отметить, что, несмотря на то что рабочие частоты большинства полупроводниковых приборов далеки от терагерцового диапазона, недавние успехи в разработке не требующих охлаждения кремниевых полевых транзисторов, а также диодов Шоттки гипервысоких частот [3], ставят задачу их дальнейшей оптимизации с помощью математического моделирования, которое ранее проводилось на основе низкочастотных локально-равновесных моделей.

Цель данной работы - формулировка непротиворечивой локально-неравновесной диффузионно-дрейфовой модели переноса носителей заряда, применимой для описания переходных ионизационных процессов в полупроводниковых структурах при воздействии импуль- сов ионизирующих излучений, длительностью порядка времени релаксации энергии и импульса электроннодырочной плазмы. В качестве исходного подхода была выбрана модель Каттанео-Вернотта [4,5], применяющаяся при описании быстропротекающих процессов тепломассопереноса, используемая в расширенной неравновесной термодинамике [6].

\section{2. Математическая модель}

Система уравнений переноса носителей заряда в диффузионно-дрейфовом приближении включает уравнение Пуассона (1) и уравнения непрерывности плотности электрического тока электронов (2) и дырок (3) [7]:

$$
\begin{gathered}
\varepsilon_{0} \nabla(\varepsilon \nabla \varphi)+q\left(N_{d}-N_{a}+p-n\right)=0, \\
\frac{\partial n}{\partial t}=\frac{\nabla j_{n}}{q}-R+G, \\
\frac{\partial p}{\partial t}=-\frac{\nabla j_{p}}{q}-R+G,
\end{gathered}
$$

где $\varepsilon$ - диэлектрическая проницаемость материала, $\varepsilon_{0}$ - диэлектрическая проницаемость вакуума, $\varphi-$ потенциал электрического поля, $q-$ элементарный заряд, $N_{d}$ и $N_{a}$ - концентрации доноров и акцепторов, $n$ и $p$ - концентрация электронов и дырок, $t-$ текущее время, $R$ и $G-$ скорости рекомбинации и генерации. Совместно с выражениями, определяющими плотность электрического тока электронов $j_{n}$ и дырок $j_{p}$, выражения (1)-(3) образуют замкнутую систему дифференциальных уравнений в частных производных.

Выражения для плотностей электрического тока электронов (4) и дырок (5) традиционной локально- 
Характерные значения скорости распространения возмущений и времени релаксации электронов для некоторых полупроводников при комнатной температуре

\begin{tabular}{c|c|c|c|c}
\hline Полупроводник & $\begin{array}{c}\text { Эффективная } \\
\text { масса [13], } m_{0}\end{array}$ & $\begin{array}{c}\text { Коэффициент } \\
\text { диффузии [13], см }{ }^{2} / \mathrm{c}\end{array}$ & $\begin{array}{c}\text { Скорость } \\
\text { распространения } \\
\text { возмущения, } \times 10^{7} \text { см/с }\end{array}$ & $\begin{array}{c}\text { Время } \\
\text { релаксации, пс }\end{array}$ \\
\hline $\mathrm{Ge}$ & 0.223 & 97.5 & 2.3 & 0.2 \\
$\mathrm{Si}$ & 0.328 & 37.5 & 1.9 & 0.1 \\
$\mathrm{GaAs}$ & 0.067 & 212.5 & 4.2 & 0.1
\end{tabular}

равновесной диффузионно-дрейфовой модели записываются в виде [7]

$$
\begin{aligned}
& j_{n}=-q \mu_{n} n \nabla \varphi+q D_{n} \nabla n, \\
& j_{p}=-q \mu_{p} p \nabla \varphi+q D_{p} \nabla p,
\end{aligned}
$$

где $\mu_{n}$ и $\mu_{p}-$ подвижности, $D_{n}$ и $D_{p}-$ коэффициенты диффузии электронов $(n)$ и дырок $(p)$.

Последовательная критика локально-равновесных моделей переноса произведена на примере уравнения теплопроводности и приведена в работах $[8,9]$. Отмечается, что закон Фурье может рассматриваться только как приближенное описание теплопроводности, в котором пренебрегается временем, необходимым для ускорения потока тепла, и предполагается мгновенное нарастание потока тепла во всех точках одновременно [10]. Для быстропротекающих процессов это приближение вступает в противоречие с принципом причинности [11].

Известно, что условия локального термодинамического равновесия сплошной среды записываются в виде [12]

$$
\begin{aligned}
& L \sim \Psi / \frac{d \Psi}{d x} \gg l, \\
& T \sim \Psi / \frac{d \Psi}{d t} \gg \tau,
\end{aligned}
$$

где $\Psi$ - макропараметр системы - потенциал переноса (для полупроводников это концентрация электронов или дырок), $\tau$ - время релаксации системы к равновесному состоянию, $v=\sqrt{a / \tau}-$ скорость распространения возмущений в среде, $a$ - коэффициент переноса (в данном случае коэффициенты диффузии электронов или дырок), $l=v \tau$ - пространственный микромасштаб, на котором происходит релаксация системы к термодинамическому равновесию, т.е. корреляционная длина, $V-$ скорость бегущей волны, $L=a / V$ - макромасштаб - характерный размер фронта бегущей волны, $T=a / V^{2}-$ характерное время изменения макропараметров системы.

Соотношения локального термодинамического равновесия (6) и (7) эквивалентны следующим неравенствам: $v \gg V$ и $T \gg \tau$, т. е. классическая локально-равновесная теория переноса справедлива, если характерная скорость данного процесса много меньше скорости распространения возмущений в среде, при этом время релаксации, в течение которого устанавливается равновесие в малых, но макроскопических областях, оказывается намного меньше характерного времени рассматриваемого процесса [12].

В случае полупроводников скорость распространения возмущений можно принять равной средней скорости движения носителей заряда. В таблице приведены характерные значения скорости распространения возмущений и времени релаксации электронов для некоторых полупроводников при комнатной температуре.

Из приведенной таблицы следует, что локальноравновесная диффузионно-дрейфовая модель становится формально неприменимой для анализа переходных ионизационных процессов в полупроводниковых структурах при воздействии тяжелых заряженных частиц и ультракоротких лазерных импульсов, длительностью менее 0.1 пс. Следует отметить, что более продвинутая квазигидродинамическая модель [14,15], учитывающая баланс энергии и импульса между электронно-дырочной подсистемой и кристаллической решеткой полупроводника в локально-равновесной постановке также становится формально некорректной для анализа быстропротекающих релаксационных процессов.

Предложенная в работах [8,9] система дифференциальных уравнений диффузии приведена в работе [16] в виде

$$
J_{i}=L_{i}^{(r)} \frac{\partial J_{i}}{\partial t}+\sum_{k=1}^{N}\left(L_{i k} X_{k}+L_{i k}^{\prime} \frac{\partial X_{k}}{\partial t}\right),
$$

где $J_{i}$ - поток среды, $X=\nabla \Psi-$ движущие силы, $L$ и $L^{\prime}$ - постоянные феноменологические коэффициенты. Если в соотношении (8) пренебречь производной от движущей силы $L_{i k}^{\prime} \frac{\partial X_{k}}{\partial t}=0$, положить $L_{i}^{(r)}=-\tau_{s c}$, $L_{i k}= \pm q D$ и добавить дрейфовое слагаемое для электронной $-q \mu_{n} n \nabla \varphi$ и дырочной $-q \mu_{p} p \nabla \varphi$ плотности тока, то получатся выражения, подобные [4,5]. Используя регуляризацию Каттанео-Вернотта $[4,5,17,18]$, можно получить следующие выражения для плотностей электронного (9) и дырочного (10) токов:

$$
\begin{aligned}
& j_{n}=-q \mu_{n} n \nabla \varphi+q D_{n} \nabla n-\tau_{s c n} \frac{\partial j_{n}}{\partial t}, \\
& j_{p}=-q \mu_{p} p \nabla \varphi-q D_{p} \nabla p-\tau_{s c p} \frac{\partial j_{p}}{\partial t},
\end{aligned}
$$

где $\tau_{s c n}$ и $\tau_{s c p}-$ среднее время между рассеяниями для электронов и дырок. 
Следует отметить, что в общем случае коэффициенты диффузии $D_{n}$ и $D_{p}$, а также подвижности $\mu_{n}$ и $\mu_{p}$ носителей заряда могут быть нелокальными и нестационарными функциями напряженности электрического поля, концентрации легирующей примеси и температуры кристаллической решетки полупроводника. Однако даже в простейшем случае постоянных коэффициентов $D_{n}, D_{p}$, $\mu_{n}, \mu_{p}$ описываемый локально-неравновесный процесс обладает инерционными свойствами: плотность тока $j$ реагирует на изменение концентрации, градиентов потенциала и концентрации не в тот же момент времени $t$, а позже, спустя время релаксации $\tau$, так как

$$
j(t+\tau)=j(t)+\tau \frac{\partial j(t)}{\partial t}=-q n \mu \nabla \varphi \pm q D \nabla n .
$$

Численный метод решения системы (1)-(5) описан в работе [19]. Учет ненулевого значения времени релаксации осуществлялся путем коррекции матрицы массы $M$ из нормированной системы дифференциально-алгебраических уравнений вида $M \frac{d u}{d t}=f(u, t)$, где $f(u, t)$ — векторная функция системы уравнений (1)-(5), $u$ - вектор неизвестных $\left(\varphi, n, p, j_{n}, j_{p}\right)$.

\section{3. Результаты и их обсуждение}

В качестве тестовой структуры был рассмотрен кремниевый диод Шоттки. Концентрация примеси $n$-типа в рабочей области принималась равной $10^{17} \mathrm{~cm}^{-3}$, длина структуры 1 мкм, высота барьера Шоттки $0.55 \mathrm{~B}$, среднее время между рассеяниями электронов и дырок 0.1 пс. На данную структуру в отсутствие внешнего приложенного напряжения моделировалось воздействие импульса ионизирующего излучения с максимальной скоростью генерации неравновесных носителей заряда $G_{0}=10^{27} \mathrm{~cm}^{-3} / \mathrm{c}$, что характерно при воздействии тяжелых заряженных частиц космического пространства, первичных атомов отдачи при воздействии атмосферных нейтронов, воздействии субпикосекундных лазерных импульсов и т.д.

Форма воздействия задавалась в двухэкспоненциальном виде

$$
G(t)=G_{0} \frac{\exp (-\alpha t)-\exp (-\beta t)}{\exp \left(-\alpha t_{0}\right)-\exp \left(-\beta t_{0}\right)},
$$

где $t_{0}=\frac{1}{\alpha-\beta} \ln \left(\frac{\alpha}{\beta}\right)$ - время, соответствующее максимальной амплитуде импульса ионизирующего излучения, $\alpha$ и $\beta-$ коэффициенты, определяющие длительность импульса, а также его переднего и заднего фронтов. Длительность импульса $\tau_{p}$ определялась по уровню амплитуды 0.5 от ее максимального значения, длительность переднего фронта импульса $\tau_{f}$ определялась по разности уровней амплитуды 0.9 и 0.1 от ее максимального значения (см. вставку на рис. 1). Отношение длительности переднего фронта импульса $\tau_{f}$

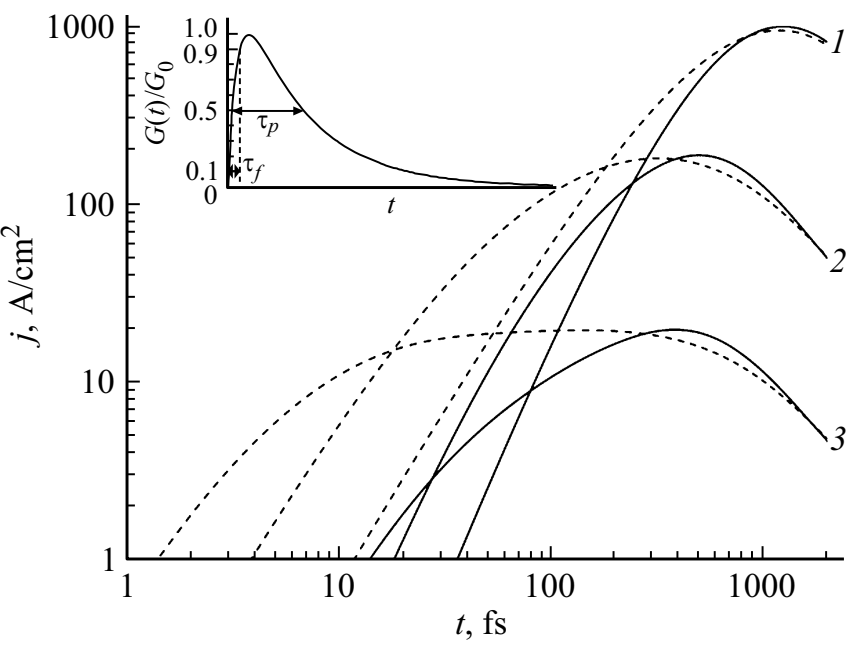

Рис. 1. Плотность фототока кремниевого диода Шоттки при воздействии импульса ионизирующего излучения длительностью: $1-1,2-0.1$ и $3-0.01$ пс; пунктирная кривая - локально-равновесная диффузионно-дрейфовая модель; сплошная кривая - локально-неравновесная диффузионнодрейфовая модель. На вставке приведена форма импульса ионизирующего излучения: $\tau_{p}$ - длительность импульса, $\tau_{f}-$ длительность переднего фронта импульса

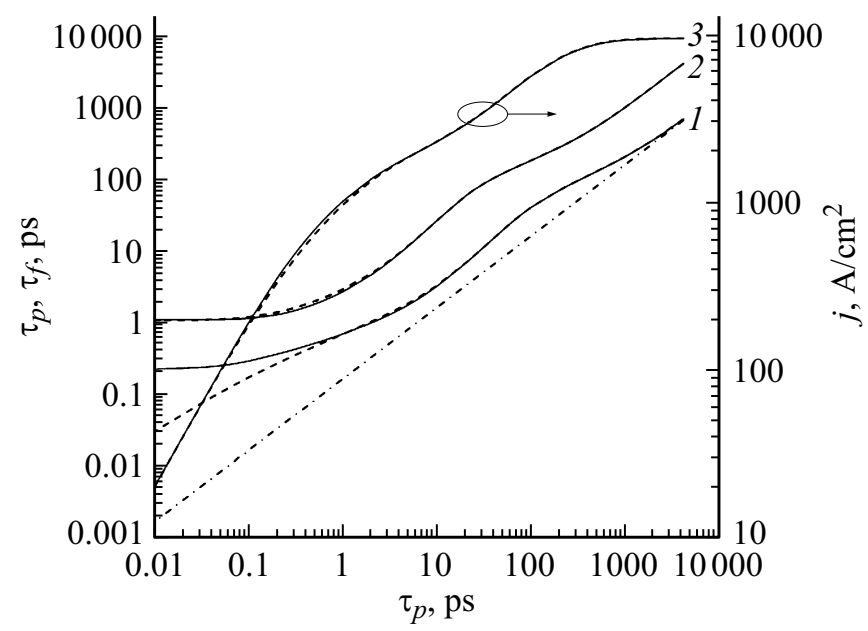

Рис. 2. Длительность переднего фронта импульса (1) и импульса фототока (2), максимальная амплитуда фототока (3) в зависимости от длительности импульса ионизирующего излучения: пунктирная кривая - локальноравновесная диффузионно-дрейфовая модель; сплошная кривая - локально-неравновесная диффузионно-дрейфовая модель; штрихпунктирная кривая - длительность переднего фронта импульса ионизирующего излучения

к длительности импульса $\tau_{p}$ в проведенных расчетах выбиралось постоянным $\tau_{f} / \tau_{p}=0.16$.

Результаты моделирования релаксации фототока приведены на рис. 1 для длительностей воздействия импульсов ионизирующего излучения $\tau_{p}=0.01,0.1,1$ пс. Из рисунка видно, что на временах выше нескольких пикосекунд результаты расчета по локально-равновесной и 

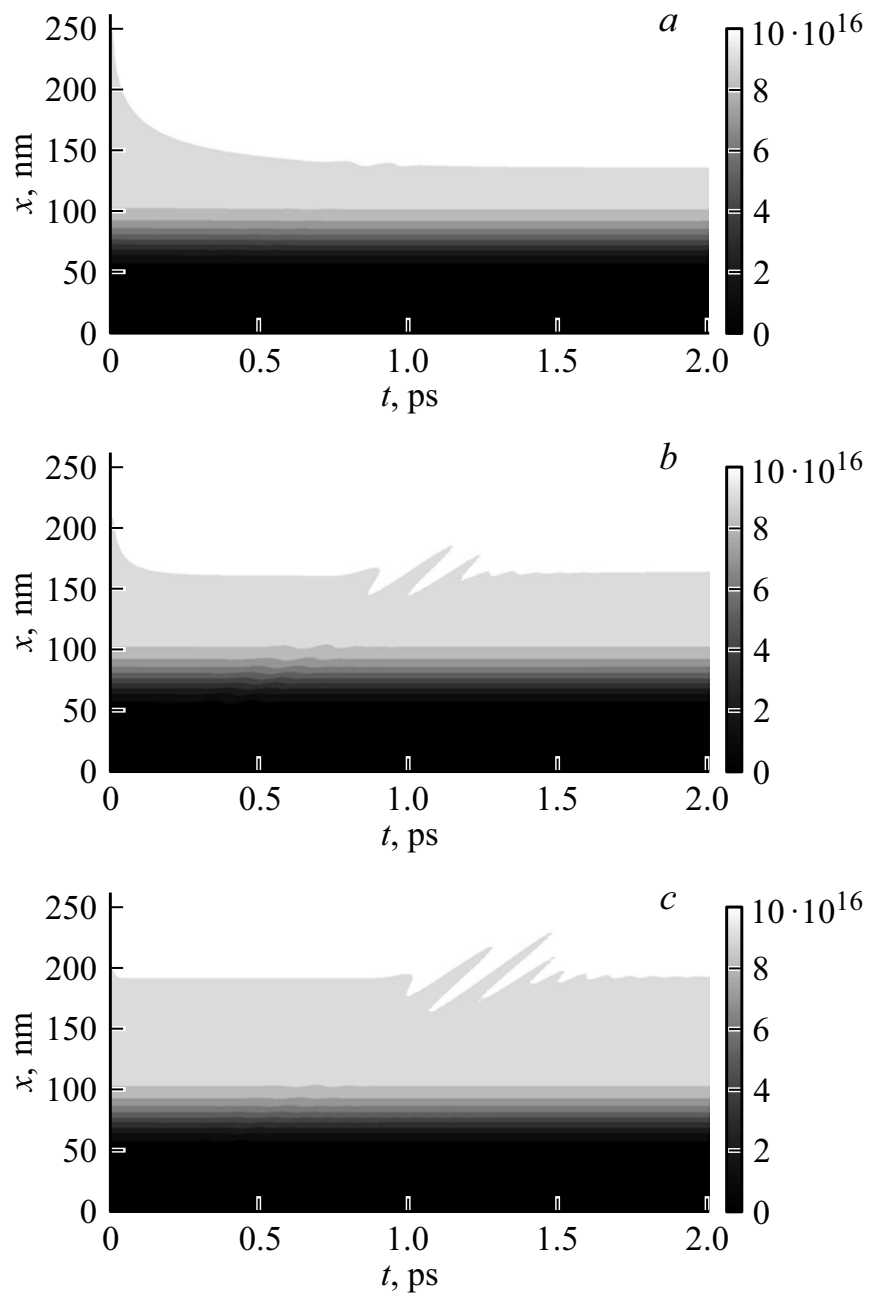

Рис. 3. Пространственно-временно́е распределение концентрации электронов в рабочей области диода Шоттки (локально-неравновесная диффузионно-дрейфовая модель) при воздействии импульса ионизирующего излучения длительностью, пс: $a-1, b-0.1, c-0.01$.

локально-неравновесной диффузионно-дрейфовым моделям совпадают. Это означает, что для описания „медленных“ по сравнению со временем релаксации процессов достаточно пользоваться традиционной локальноравновесной диффузионно-дрейфовой моделью.

На рис. 2 приведены зависимости длительностей переднего фронта импульса и самого импульса, а также максимальной амплитуды фототока от длительности импульса ионизирующего излучения $\tau_{p}$, которая варьировалась в пределах 0.01-4000 пс. Видно, что передний фронт импульса фототока, рассчитанный при помощи локально-равновесной модели, значительно короче, чем для локально-неравновесной модели. Это объясняется аномально высокой скоростью движения носителей заряда, существенно превосходящей баллистический предел, что, очевидно, нефизично. На временах, в несколько раз превосходящих время релаксации, скорости потоков носителей заряда выравниваются, что приводит к прак- тически идентичному заднему фронту импульса, рассчитанному на основе обеих моделей. Длительность заднего фронта импульса существенно больше длительности переднего фронта импульса, поэтому длительность отклика на воздействие ионизирующего излучения практически не зависит от применяемой при расчетах модели: локально-равновесной или локально-неравновесной.

Пространственно-временно́е распределение концентрации электронов в рабочей области диода Шоттки приведено на рис. 3. Конечная скорость распространения носителей заряда в полупроводниках, ограниченная баллистическим пределом, приводит к образованию волн электронов в самосогласованном электрическом поле. Амплитуда этих волн определяется скоростью нарастания концентрации неравновесных носителей заряда, т. е. параметрами воздействующего импульса ионизирующего излучения.

Таким образом, получено, что при длительностях импульса ионизирующего излучения, существенно больших времени релаксации возбуждений в электроннодырочной плазмы, локально-равновесная и локальнонеравновесная диффузионно-дрейфовые модели дают идентичный результат. С уменьшением длительности импульса ионизирующего излучения до долей пикосекунд начинает проявляться инерционность электроннодырочной плазмы, что приводит к более медленному нарастанию переднего фронта импульса фототока, рассчитанного в рамках локально-неравновесного приближения, по сравнению с безынерционной локальноравновесной моделью. Дальнейшее уменьшение длительности импульса ионизирующего излучения приводит к появлению бегущих волн концентрации электронов, возникающих в обедненной области и затухающих в толще полупроводника.

\section{4. Заключение}

Результаты проведенных расчетов показывают, что длительность переднего фронта импульса фототока перестает зависеть от длительности импульса ионизирующего излучения при ее уменьшении до времени релаксации электронно-дырочной плазмы. Это может оказаться важным при анализе сбоев и отказов высокочастотных элементов, работающих в условиях воздействия тяжелых заряженных частиц космического пространства.

Другим возможным применением предложенной локально-неравновесной диффузионно-дрейфовой модели в приближении Каттанео-Вернотта является расчет характеристик умножителей частот субмиллиметрового диапазона на диодах Шоттки.

Дальнейшее развитие локально-неравновесной модели переноса носителей заряда в полупроводниковых приборах может вестись по следующим направлениям: формулировка локально-неравновесной квазигидродинамической модели, включающей помимо уравнений непрерывности плотности электрического тока также уравнения 
непрерывности потоков энергии подвижных носителей заряда, и формулировка локально-неравновесной модели в приближении Гюера-Крумхансля, аналогичном применяемому в теории тепломассопереноса $[6,10,20,21]$.

Работа выполнена при поддержке программ РАН, гранта РФФИ № 15-02-07935.

\section{Список литературы}

[1] R. Jones, A.M. Chugg, C.M.S. Jones, P.H. Duncan, C.S. Dyer, C. Sanderson. IEEE Trans. Nucl. Sci., 47 (3), 539 (2000).

[2] А.И. Чумаков, А.Н. Егоров, О.Б. Маврицкий, А.В. Яненко. Микроэлектроника, 33 (2), 137 (2004).

[3] M. Sakhno, A. Golenkov, F. Sizov. J. Appl. Phys., 114 (16), 164503 (2013)

[4] P. Vernotte. Comptes Rendus Acad. Sci. Paris, 246, 3154 (1958).

[5] C. Cattaneo. Comptes Rendus Acad. Sci. Paris, 247, 431 (1958).

[6] D. Jou, J. Casas-Vazquez, G. Lebon. Extended irreversible thermodynamics (Spinger, 2010).

[7] W. Shockley. Bell Syst. Techn. J., 28 (7), 435 (1949).

[8] L. Onsager. Phys. Rev., 37 (4), 405 (1931).

[9] L. Onsager. Phys. Rev., 38 (12), 2265 (1931).

[10] К.В. Жуковский. Уч. записки физ. фак. МГУ, 6 (4), 1740301 (2017).

[11] М.Ю. Белевич. Уч. записки росс. гос. гидрометеоролог. унта, 10, 101 (2009).

[12] С.Л. Соболев. УФН, 161 (3), 5 (1991).

[13] С.М. Зи. Физика полупроводниковых приборов (М., Мир, 1985).

[14] R. Stratton. Phys. Rev., 126 (6), 2002 (1962).

[15] K. Blotekjaer. IEEE Trans. Electron Dev., 17 (1), 38 (1970).

[16] А.В. Лыков. Теория теплопроводности (М., Высш. шк., 1967).

[17] M. Lundstrom. Fundamentals of carrier transport (Cambridge University Press, 2000).

[18] F. Ekoue, A. Fouache d'Halloy, D. Gigon, G. Plantamp, E. Zajdman. World Academy of Science, Engineering and Technology Int. J. Phys. Mathemat. Sci., 7 (5), 772 (2013).

[19] В.К. Киселев, С.В. Оболенский, А.С. Пузанов, А.В. Скупов. РЭ, 17 (2), 10 (2014).

[20] R.A. Guyer, J.A. Krumhansl. Phys. Rev., 148 (2), 766 (1966).

[21] R.A. Guyer, J.A. Krumhansl. Phys. Rev., 148 (2), 778 (1966).

Редактор Г.А. Оганесян

\author{
A.S. Puzanov' ${ }^{1}$, S.V. Obolenskiy', V.A. Kozlov ${ }^{1,2}$ \\ ${ }^{1}$ Lobachevski University of Nizhny Novgorod, \\ 603600 Nizhny Novgorod, Russia \\ 2 Institute for Physics of Microstructure, Russian \\ Academy of Sciences, \\ 607680 Nizhny Novgorod, Russia
}

\begin{abstract}
The process of relaxation of excitations in electronhole plasma under the influence of ionizing radiation with duration, less time of relaxation of energy and momentum of mobile charge carriers is considered. On the example of calculation of transient ionization processes in silicon Schottky diode of hyperhigh frequencies the comparison of local-equilibrium and local-nonequilibrium models of charge carriers transfer is carried out. It is shown that the locally nonequilibrium model has a wider scope of applicability for the description of fast-flowing relaxation
\end{abstract} processes.

\section{Application of a locally nonequilibrium diffusion-drift Cattaneo-Vernotte model for calculating the relaxation of the photocurrent in diode structures under the influence of subpicosecond pulses of ionizing radiation}

